\title{
Analysis of the power dependent tuning of a varactor-loaded metamaterial at microwave frequencies
}

\author{
Da Huang, Ekaterina Poutrina, and David R. Smith ${ }^{\text {a) }}$ \\ Department of Electrical and Computer Engineering, Center for Metamaterials and Integrated Plasmonics, \\ Pratt School of Engineering, Duke University, P.O. Box 90291, Durham, North Carolina 27708, USA
}

(Received 16 January 2010; accepted 11 February 2010; published online 11 March 2010)

\begin{abstract}
We present an analysis of the nonlinear, power-dependent resonance frequency shift for two metamaterial mediums consisting of arrays of varactor-loaded split ring resonators (VLSRRs). We confirm that, over a limited range of power, a VLSRR medium can be described by its second and third order nonlinear susceptibilities, making it a useful analog medium for the quantitative investigation of other nonlinear phenomena that might be achieved using inherently nonlinear materials integrated into metamaterials. Experimental measurements of the resonance frequency shift with power from fabricated VLSRR samples are found to be in excellent agreement with the analytical model. (C) 2010 American Institute of Physics. [doi:10.1063/1.3356223]
\end{abstract}

Metamaterials are frequently composed of planar, conducting circuits periodically patterned on dielectric substrates. By adjusting the circuit properties-such as inductance, capacitance, or resistance- the subwavelength circuits can be engineered to exhibit a wide range of effective electromagnetic properties. Resonant metamaterial structures, such as split-ring resonators (SRRs) ${ }^{1}$ or electric coupled resonators (ELCs) ${ }^{2}$ have been explored as the basis for electric or magnetic metamaterials. Because the local electric fields are strongly enhanced in resonant metamaterials, it has been suggested that these large local fields can drive nonlinear response in materials integrated into the strong field regions. ${ }^{3}$ Alternatively, nonlinear circuit components, such as diodes, also have the potential to form nonlinear metamaterial systems. The inclusion of nonlinear components is a natural extension to the commonly used circuit description of metamaterials, although actual packaged components are likely to function reliably only at relatively low frequencies. Moreover, such components display extremely strong nonlinearities of all orders, so that the material description of a metamaterial integrated with nonlinear components will be generally complicated compared with conventional nonlinear materials. Nevertheless, over limited ranges of excitation powers, it can be expected that lower order nonlinear terms dominate, allowing the composite metamaterial to be described in a manner analogous to a nonlinear crystal. To emphasize this analogy, we refer to the structures described here as metacrystals.

Nonlinear phenomena, such as resonant frequency tuning, ${ }^{4,5}$ second harmonic generation, ${ }^{6}$ and bistability, ${ }^{4,7}, 8$ have been observed in either metacrystal composites or in single metamaterial unit cells. These initial experimental verifications of nonlinear properties have shown considerable promise; yet, there remains a need for more quantitative models of nonlinear metacrystals to be developed. The varactor-based nonlinear metacrystal provides a good starting point for further investigation, since the structures are easily fabricated and the electronic circuit properties of both the metamaterial and the integrated components are known with great precision.

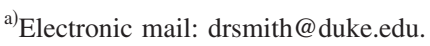

In the present work, we form a nonlinear metacrystal at microwave frequencies by integrating packaged varactors into the capacitive gaps of SRRs. The metacrystal comprises the same sort of varactor-loaded SRRs (VLSRRs) explored by Wang et $a l^{4}{ }^{4}$ We investigate both single-gap and the double-gap SRR-based mediums, in the latter case with varactors placed in a back-to-back configuration, as shown in Figs. 1(a) and 1(b). The equivalent circuit $\operatorname{model}^{4}$ for a single-gap unit cell is presented in Fig. 1(c). The back-toback configuration eliminates the dissipative current that otherwise runs through the circuit at high driving fields in the single-gap VLSRR. Here, we use the shift of the resonance frequency of the VLSRR with increasing incident power as a means of characterizing the nonlinear response of the composite metacrystal. The frequency shift can be directly related to the third order nonlinear susceptibilities associated with the composite medium. ${ }^{9}$ Our goal is to provide a quantitative comparison between the theoretical prediction for the resonant frequency shift and the experimental results, and to discuss the range of validity of the small order expansion.

To form the nonlinear VLSRR, we implement the Skyworks SMV1231 varactor, which has a capacitance of the form $C\left(V_{D}\right)=C_{0}\left(1-V_{D} / V_{P}\right)^{-M}$. Here $V_{D}$ is the bias voltage, $C_{0}=2.4 \mathrm{pF}$ is the zero bias capacitance, $M=0.8$ is the gradient coefficient of the varactor, and $V_{P}=1.5 \mathrm{~V}$ is the intrinsic potential. Following the derivation of Ref. 4, for small amplitudes of the exciting field (and hence small voltages), the voltage on the varactor can be represented as a function of the accumulated charge $Q_{D}=\int_{V_{D}} C\left(V_{D}\right) d V_{D}$ and expanded in a Taylor series as $V_{D}(q) \approx q+a q^{2}+b q^{3}$, where $a \equiv-M / 2 V_{P}$ $\approx-0.2667, b \equiv M(2 M-1) / 6 V_{P}^{2} \approx 0.0356$, and $q$ is the renormalized voltage, $q \equiv Q_{D} / C_{0}$. The expression for $V_{D}$ can then

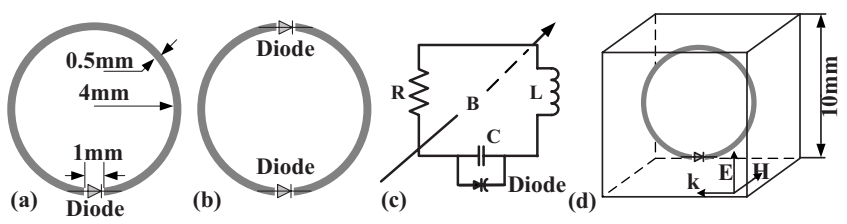

FIG. 1. (a) Single varactor loaded SRR unit. (b) Double varactor loaded SRR unit. (c) The equivalent circuit model for a single-gap unit. (d) Orientation of unit cell. 
be used in the voltage equation representing the SRR as an effective $R L C$ circuit driven by an external excitation to yield

$$
\frac{d q^{2}}{d t^{2}}+\gamma \frac{d q}{d t}+\omega_{0}^{2} q+\alpha q^{2}+\beta q^{3}=-\omega_{0}^{2} \epsilon
$$

In Eq. (1), $\alpha \equiv a \omega_{0}^{2}, \beta \equiv b \omega_{0}^{2}, \omega_{0}$ is the zero-bias resonant frequency of the effective circuit, and $\epsilon$ is the amplitude of the driving voltage resulting from the electromagnetic field incident on the unit cell.

Based on the perturbation method introduced in Ref. 10, the nonlinear susceptibility can be expressed in the form of a power series expansion in the strength of the perturbation. While a detailed derivation will be presented elsewhere, ${ }^{9}$ we summarize the results here as a means to analyze the resonant frequency tuning mechanism.

The resonance frequency shift as a function of the intensity of the applied field can be expressed as a result of a third-order self-phase-modulation process, leading to an intensity-dependent refractive index. Utilizing the voltage dependent capacitance described above in the SRR circuit model, and assuming a linearly polarized incident field $H(t)=H \exp (-i \omega t)$, we obtain the following expression for the third order nonlinear susceptibility as a function of response frequency $\omega:^{9}$

$$
\chi_{1111}^{(3)}(\omega)=\frac{F \omega_{0}^{6} \omega^{4} A^{2} \mu_{0}^{2}}{D^{3}(\omega) D(-\omega)}\left(\frac{4 a^{2} \omega_{0}^{2}}{3 D(0)}+\frac{2 a^{2} \omega_{0}^{2}}{3 D(2 \omega)}-b\right),
$$

where $F$ is the oscillator strength in the numerator of the linear SRR effective susceptibility, ${ }^{11} A$ is the area enclosed by the SRR structure, and $D(\omega) \equiv \omega_{0}^{2}-\omega^{2}-i \gamma \omega$ is the Lorentz function. Equation (2) enables the description of the metacrystal as a continuous nonlinear crystal with an anisotropic, power-dependent permeability given by

$$
\mu_{\mathrm{eff}}^{N L}(\omega)=1+\frac{F \omega^{2}}{D(\omega)}+3 \chi_{1111}^{(3)}|H|^{2},
$$

where $\chi_{1111}^{(3)}$ is given by Eq. (2). At low or zero (H $=0 \mathrm{~A} / \mathrm{m}$ ) power levels, Eq. (3) reverts to the standard linear Lorentzian form. However, both the model described by Eq. (1) and the series expansion of the nonlinear magnetization used in deriving Eq. (3) are applicable at low power levels. By examining the fourth and higher-order terms in the series expansion, ${ }^{9}$ we estimated the maximum applicable $H$ in Eq. (3) to be close to $27 \mathrm{~mA} / \mathrm{m}$.

The resonant frequency of the medium appears as the minimum in the transmission coefficient of a plane wave propagating through the metacrystal. As the incident power increases, the minimum shifts indicating the powerdependent change in the resonant frequency. We verify the validity of Eqs. (2) and (3) in the range of the field amplitudes discussed above by comparing the transmission curves of a single-layer metacrystal slab at varying incident power levels, obtained analytically based on Eq. (3) and from the measurements, as discussed below.

For the analytical calculation of the transmission, we first calculate the $\mu_{\text {eff }}^{N L}$ using a full wave simulator (Microwave Studio, CST), modeling the varactor under zero bias. Applying a standard retrieval procedure, we can extract the following parameters: $F=0.1426, \omega_{0}=5.14 \times 10^{9} \mathrm{rad} / \mathrm{s}, \gamma$ $=1.6022 \times 10^{8} \mathrm{~s}^{-2} \mathrm{~V}^{-1}$ for the single-varactor metacrystal.

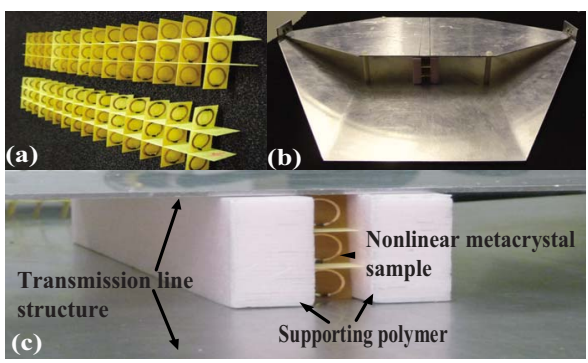

FIG. 2. (Color online) (a) VLSRR metacrystal sample. (b) Transmission line structure. (c) Sample in waveguide testing assemble.

The other parameters are as indicated in Fig. 1; the thickness of the FR4 PCB substrate is $0.2 \mathrm{~mm}$. We then apply Eq. (3) to calculate the transmission coefficient of a single-layer metacrystal slab with permittivity $\epsilon_{\text {eff }}=1.63$ using the transfer matrix method. ${ }^{11}$ The resulting transmittance at different field intensity levels is shown in Fig. 3(b). According to the analytical result, the resonant frequency of the single VLSRR medium shifts downward as the excitation field intensity increases.

For the double-varactor medium, the signs of the bias voltages across the two varactors are opposite due to their back-to-back configuration, leading to the condition $V_{d 1}(q)=-V_{d 2}(-q), V_{d 1}$, and $V_{d 2}$ being the voltages across the varactors. As a result, there is a cancellation of the evenorder terms in the Taylor expansion of $V_{d 1}+V_{d 2}$ in Eq. (1), and hence no contribution to $\chi_{1111}^{(3)}$ from the second-order susceptibility. Equation (2) thus becomes ${ }^{9} \chi_{111}^{(3)}$ $=F \omega_{0}^{6} \omega^{4} A^{2} \mu_{0}^{2}(-2 b) / D^{\prime 3}(\omega) D^{\prime}(-\omega)$, where $D^{\prime}(\omega)=\omega_{r}^{2}-\omega^{2}$ $-i \gamma \omega$, and $\omega_{r}=1.215 \times 2 \pi \mathrm{rad} / \mathrm{s}$ is the new resonant frequency of the circuit. The fitting parameters for the linear permeability of the double-varactor medium, as determined from Microwave Studio simulations, are $F=0.1362, \omega_{r}$ $=7.65 \times 10^{9} \mathrm{rad} / \mathrm{s}, \quad \gamma=2.953 \times 10^{8} \mathrm{~s}^{-2} \mathrm{~V}^{-1}$, and $\omega_{0}$ is the resonant frequency from the single-gap case. The opposite sign of $\chi_{1111}^{(3)}$ in Eq. (3) compared to the single-gap VLSRR medium implies a resonant frequency shift opposite to that of the double-gap sample as a function of increasing excitation power. The opposite direction of the frequency shift is in fact observed in the experiments for the double-gap VLSRR medium, as discussed below. From examining the higher order terms in the series expansion for the double-gap case, the maximum applicable $H$ for up to the third order expansion in field magnitude is estimated to be about $100 \mathrm{~mA} / \mathrm{m}$. The larger validity range is expected in this case since the higherorder effective permeability terms decrease more rapidly in the absence of the contributions from the even orders.

To confirm the analytical results, VLSRR metacrystals were constructed with the same parameters as used in the above analysis. We used a single layer, $3 \times 15$ elements material, for both the single-and double varactor cases. The metacrystal samples of the two kinds are shown in Fig. 2(a). A transmission line supporting TEM wave propagation below $2 \mathrm{GHz}^{12}$ was used to measure the transmittance through the metacrystal sample, as shown in Figs. 2(b) and 2(c). An Agilent vector network analyzer (PNA N5230A) was used to launch microwaves into the transmission line and detect the transmitted fields. The frequency-dependent transmission properties associated with both the waveguide and connecting cables were removed using a standard calibration 

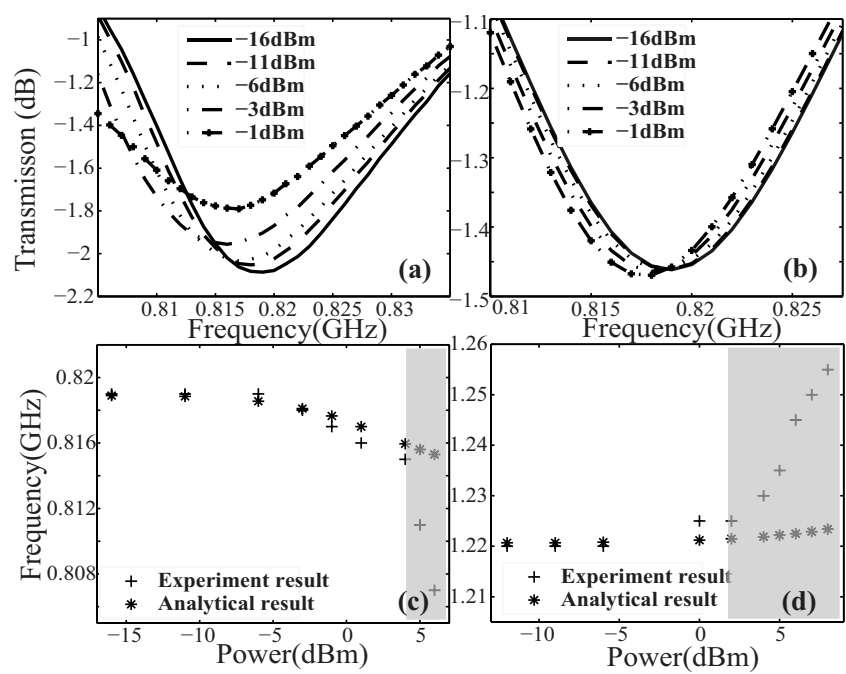

FIG. 3. (a) Experimental and (b) theoretical transmission spectrum for single varactor metacrystal. [(c) and (d)] Comparison of analytical and experimental resonant frequency shift for single- (c) and double-gap (d) metacrystals.

method. The excitation power $P_{w g}$ from the network analyzer spanned the range from -10 to $+15 \mathrm{dBm}$. The total loss from transmission line structure, connection cable, and adapter was measured to be about $6 \mathrm{~dB}$, so that the actual power exciting the sample ranged from -16 to $9 \mathrm{dBm}$. The corresponding magnetic field intensity can be found as $H$ $=\sqrt{P_{w g} / 2 \eta S_{0}}$, where $\eta=377 \Omega$ is the free space impedance and $S_{0}=45 \mathrm{~cm}^{2}$ is the transmission line cross section area. According to these expressions, the incident field ranges from 3 to $68 \mathrm{~mA} / \mathrm{m}$, which includes the field range considered in the analytical calculation. We note however that the actual power inside the waveguide and the corresponding magnetic field are accurate up to about $2 \mathrm{dBm}$ due to the imperfect loss measurement and the approximate mode area used in the calculation.

In agreement with the analytical predictions, the resonant frequency of the single-gap VLSRR metacrystal shifts downwards with increasing power, as seen in Fig. 3(a). The comparison of the analytical and experimental resonant frequencies as functions of incident power are presented in Figs. 3(c) and 3(d) for the single- and double-gap metacrystals. For the single-varactor case, good agreement between theory and measurements is found up to a power of about $4 \mathrm{dBm}(H=27 \mathrm{~mA} / \mathrm{m})$. This value agrees well with the anticipated validity range of the analytical theory. For the double-varactor medium, the resonant frequency shifts upwards, as expected from the theory. Good agreement between theory and measurements is again observed, up to about $2 \mathrm{dBm}(H \approx 21 \mathrm{~mA} / \mathrm{m})$, which is smaller than the theoretically predicted validity range. We attribute this discrepancy to the fact that the actual double-varactor unit cells are not ideally symmetric, leading to a nonideal cancellation of the even-order terms in the voltage expansion. The contribution from these terms becomes pronounced once the field strength increases, producing a stronger resonance shift. We also note that, for the single-gap metacrystal, the experimental resonance curves broaden while the amplitudes of the resonances are reduced with increasing power. The damping of the resonance results from the dissipative current running through the varactor, ${ }^{4}$ which is not accounted in the analytical model and therefore is not present in the analytical curves.

The comparison of the analytical and experimental transmission curves demonstrates that the varactor loaded nonlinear metacrystals can be described by their effective second and third order nonlinear susceptibilities, for values of the excitation magnetic field magnitude up to 27 and $21 \mathrm{~mA} / \mathrm{m}$ for the single-and double-varactor metacrystals, respectively. The results point to the feasibility of nonlinear metamaterials formed by integrating inherently nonlinear crystals into the high-field regions of metamaterial elements. In addition, the microwave nonlinear medium investigated here should serve as a useful analog medium for the demonstration of a host of other nonlinear metacrystal phenomena.

We thank Dr. Stéphane Larouche for helpful discussions and suggestions. This work was supported by the Air Force Office of Scientific Research (Contract No. FA9550-09-10562).

${ }^{1}$ J. Pendry, A. Holden, D. Robbins, and W. Stewart, IEEE Trans. Microwave Theory Tech. 47, 2075 (1999).

${ }^{2}$ D. Schurig, J. J. Mock, and D. R. Smith, Appl. Phys. Lett. 88, 041109 (2006).

${ }^{3}$ A. A. Zharov, I. V. Shadrivov, and Y. S. Kivshar, Phys. Rev. Lett. 91, 037401 (2003).

${ }^{4}$ B. Wang, J. Zhou, T. Koschny, and C. M. Soukoulis, Opt. Express 16, 16058 (2008).

${ }^{5}$ I. V. Shadrivov, A. B. Kozyrev, D. W. van der Weide, and Y. S. Kivshar, Opt. Express 16, 20266 (2008).

${ }^{6}$ Z. Wang, Y. Luo, L. Peng, J. Huangfu, T. Jiang, D. Wang, H. Chen, and L. Ran, Appl. Phys. Lett. 94, 134102 (2009).

${ }^{7}$ D. A. Powell, I. V. Shadrivov, and Y. S. Kivshar, Appl. Phys. Lett. 91, 144107 (2007).

${ }^{8}$ N. M. Litchinitser, I. R. Gabitov, and A. I. Maimistov, Phys. Rev. Lett. 99, 113902 (2007).

${ }^{9}$ E. Poutrina, D. Huang, and D. R. Smith (to be published).

${ }^{10}$ R. W. Boyd, Nonlinear Optics, 3rd ed. (Academic, Amsterdam, 2008).

${ }^{11}$ D. R. Smith, D. C. Vier, T. Koschny, and C. M. Soukoulis, Phys. Rev. E 71, 036617 (2005).

${ }^{12}$ B.-I. Popa and S. A. Cummer, Phys. Rev. Lett. 100, 207401 (2008). 